\title{
Analyse de l'effet de la concurrence végétale sur la croissance du Moabi en forêt dense tropicale perturbée de l'Est - Cameroun
}

\author{
MBONAYEM LIBOUM ${ }^{1,2^{*}}$ et K.S. BOBO ${ }^{1,3}$ \\ ${ }^{1}$ Université de Dschang, Faculté d'Agronomie et des Sciences Agricoles, \\ Département de Foresterie, B.P.: 222 Dschang, Cameroun. \\ ${ }^{2}$ Centre Technique de la Forêt Communale (CTFC), BP. : 15107 Yaoundé, Cameroun. \\ ${ }^{3}$ Laboratoire de Faune, Aires Protégées, Sylviculture et Technologie du Bois (LAFAPSYTEB), \\ Université de Dschang, B.P. : 222 Dschang, Cameroun. \\ *Auteur correspondant ; E-mail: libyem@gmail.com; Tel: +237674226197
}

\section{RESUME}

L'une des composantes clé de la dynamique des forêts est la croissance des espèces qui est à ce jour peu maîtrisée. L'objectif de la présente étude est de maîtriser les variables de compétition qui décrivent le mieux la trajectoire de croissance du Moabi en peuplement hétérogène. Vingt placettes semi permanentes circulaires de $20 \mathrm{~m}$ de rayon centrées sur chacun des 60 arbres étudiés ont été mises en place par la Société Pallisco depuis 2008. Seul l'indice de surface terrière relative du peuplement pondérée par la distance avec l'arbre considéré explique le mieux l'accroissement en diamètre et l'accroissement en surface terrière de manière soutenue. La combinaison prédisant le mieux l'accroissement diamétrique du Moabi comporte trois variables dont deux indices indépendants des distances, et un dépendant des distances et correspondant au diamètre cumulé des arbres du peuplement pondéré par la distance avec l'arbre considéré.En définitive, au-delà des indices de concurrence indépendants et dépendants de la distance, le diamètre moyen des arbres et l'indice d'éclairement du houppier peuvent rentrer dans l'élaboration d'un modèle prédictif efficient de l'accroissement en diamètre et en surface terrière en vue de planifier l'exploitation durable de cette essence.

(C) 2017 International Formulae Group. All rights reserved.

Mots clés: Accroissement en diamètre, compétition végétale, indices de concurrence, modèle de croissance, prédictibilité de la croissance.

\section{Analysis of effect of plant competition on the growth of Moabi in dense tropical disturbed forest of Eastern-Cameroon}

\begin{abstract}
One of the key components of forest dynamic is the species growth which is less mastered today. The aim of the present study was to master competitive variables that best describe the growth pattern of Moabi in an heterogenous stand. Twenty semi-permanent circular plots of $20 \mathrm{~m}$ radius centred on each of the 60 trees studied were established by the Pallisco Company in 2008. Only the stand relative basal area index pondered with the distance to a reference tree best explain the increase in diameter and basal area in a sustainable
\end{abstract}


manner. The combination that best predict the increase in diameter of Moabi is composed of three variables of which two indices independent of the distances, and one that depends on the distances and which corresponds to the cumulated diameter of trees from the stand pondered by the distance to a reference tree. In all, apart from the indices independent and dependent of the distances, the mean diameter of trees and the canopy light index can be considered in the elaboration of an efficient predictive model of the increase in diameter and in basal area when planning the sustainable exploitation of the species.

(C) 2017 International Formulae Group. All rights reserved.

Keywords: Competitive indices, growth model, growth prediction, increase in diameter, vegetation competition.

\section{INTRODUCTION}

Les forêts constituent un réservoir de biodiversité et jouent un rôle fondamental dans la satisfaction des besoins de base des communautés locales (Agbo et al., 2017). La relation entre la biodiversité et le fonctionnement des écosystèmes est une question écologique fondamentale (Dibong et Ndjouondo, 2014). L'étude des phénomènes de concurrence en forêt tropicale humide s'insère dans un cadre plus large de recherche sur les mécanismes qui régissent le fonctionnement de cet écosystème, afin de donner aux gestionnaires forestiers les moyens d'intervenir plus rationnellement. En effet, il est beaucoup plus difficile d'avoir une connaissance scientifique dans le domaine des forêts mélangées, et l'étude des forêts hétérogènes est un sujet difficile d'écologie (Franc, 2008). Ainsi, pour la planification des ressources forestières, les modèles de croissance apportent aux gestionnaires les éléments qui leur font souvent défaut. La caractérisation d'un peuplement forestier, qui comporte plusieurs espèces et des arbres de tous les âges et de toutes les dimensions, par des variables globales telles que la hauteur dominante ou la surface terrière, n'a guère de sens, et il va de soi que des modèles de croissance plus détaillés que les modèles de peuplement (tables de peuplement) sont alors nécessaires pour prédire la croissance (Picard, 2007). Dès lors, les chercheurs s'intéressent depuis longtemps à la prédiction de l'évolution des peuplements forestiers, et s'attèlent à mettre au point des outils pour aider les gestionnaires forestiers (Pardé et Bouchon, 1988; Goreaud et al., 2005).
Pour concilier les aspects environnementaux et économiques de gestion des forêts tropicales, des stratégies de gestion responsable (aménagement forestier) ont été développées, et les forêts continuent de se dégrader (FAO, 2011). En effet, l'aménagement des forêts de production du bassin du Congo utilise encore des outils peu adaptés (paramètres de détermination de la dynamique des peuplements) pour définir le type de sylviculture à appliquer alors que la prédiction de l'état futur d'un peuplement hétérogène devrait passer par l'établissement de modèles de croissance en grosseur ou en hauteur des espèces en présence qui dépendent le plus souvent de la localisation de l'arbre central, son statut et l'importance de la végétation dans laquelle il évolue (Goreaud et al., 2005).

Le principe d'exclusion par la compétition souvent invoqué en écologie des communautés stipule que, dans certaines conditions (aucun avantage démographique pour les espèces rares, environnement constant dans le temps et dans l'espace, temps suffisamment long pour permettre la compétition, etc.), une espèce devient dominante en excluant toutes les autres (Blanc et al., 2003) et par conséquent croît mieux. Ainsi, peu d'espèces de forêts hétérogènes ont à ce jour fait l'objet de simulation de leur croissance individuelle en intégrant des paramètres environnementaux à des fins de gestion. Le cas du Moabi, espèce phare des forêts du Sud-Est Cameroun (Doucet et al., 2007) exploitées pour son bois, et pour laquelle la croissance a pourtant fait l'objet de plusieurs études, mérite d'être traité car très 
peu de connaissances existent sur la compétition végétale sur cette espèce dans son milieu naturel et dans un milieu perturbé. Si les données de croissance moyenne du Moabi sont disponibles, les facteurs de variation de la croissance ne sont à ce jour pas connus. La présente étude permettra de contribuer à une meilleure compréhension des variations de la croissance du Moabi en fonction des interactions de compétition avec le peuplement environnant, données utiles dans la simulation de la dynamique de croissance du peuplement à des fins de planification de la gestion durable des forêts car la compétition intervient dans de très nombreux modèles de croissance et de dynamique forestière. Globalement, l'objectif de cette étude est d'analyser l'effet de la concurrence végétale sur la croissance du Moabi. Plus spécifiquement, il s'est agit de déterminer les paramètres de croissance et les différents indices de compétition pertinents utilisés en écologie pour décrire la concurrence du Moabi avec les arbres voisins pour l'accès aux ressources vitales ; d'analyser les variations de la croissance du Moabi et de déterminer des variables prédictives potentielles de modélisation de la croissance du Moabi.

\section{MATERIEL ET MEHODES \\ Zone d'étude}

L'étude est conduite dans une concession constituée de cinq Unités Forestières d'Aménagement (UFA 10-030, $10-039,10-041,10-042$ et 10-044) avec une superficie totale d'environ 342.000 ha. Sur le plan administratif, elle est située dans la région de l'Est, département du Haut-Nyong, à cheval sur les arrondissements de Lomié, Messok et Mindourou. Sur le plan géographique, la concession est comprise entre $3^{\circ}$ et $3^{\circ} 45^{\prime}$ de latitude Nord et entre $13^{\circ} 20^{\prime}$ et $14^{\circ} 32^{\prime}$ de longitude Est (feuillet cartographique $1 / 200.000^{\mathrm{e}} \quad \mathrm{NA}-33-\mathrm{XXI}$ Medoum-Institut National de Cartographie (INC) (Figure 1).
Caractéristiques climatiques de la zone d'étude

Les concessions sont soumises dans son ensemble à l'influence d'un climat équatorial chaud et humide de type guinéen classique à deux saisons de pluies entrecoupées de deux saisons sèches (Feteke et al., 2004). Au cours de l'année, les saisons se succèdent de la manière suivante :

- la petite saison des pluies de mi-mars à juin ;

- la petite saison sèche de juin à mi-août ;

- la grande saison des pluies de mi-août à mi-novembre ;

- la grande saison sèche de mi-novembre à mi-mars.

La température moyenne de la région oscille autour de $24{ }^{\circ} \mathrm{C}$. Les températures mensuelles les plus basses sont relevées au mois de juillet $\left(22,8{ }^{\circ} \mathrm{C}\right.$ à Lomié) et les plus élevées au mois d'avril $\left(24,6{ }^{\circ} \mathrm{C}\right.$ à Lomié). Les précipitations annuelles moyennes se situent le plus souvent entre 1550 et $2000 \mathrm{~mm}$ (hauteur moyenne mensuelles de pluies à Lomié au cours des 25 dernières années : $1654 \mathrm{~mm}$ ). Les maxima de précipitations sont enregistrées en avril - mai et en septembre octobre (Feteke et al., 2004).

\section{Caractéristiques pédologiques de la zone d'étude}

Du point de vue pédologique, les sols rencontrés dans les cinq UFA dérivent de roches métamorphiques. Sur la terre ferme, ce sont principalement des sols ferralitiques rouges ou jaunes typiques. Ils sont en général profonds, argileux, meubles, perméables, et présentent souvent peu d'humus. Leur teneur en bases échangeables est faible. Ce sont des sols pauvres, acides et fragiles de type Oxisols argileux à limono-argileux. Il est possible d'observer à certains endroits la cuirasse ferrugineuse d'épaisseur variable en affleurement. Dans les bas-fonds, on trouve des sols hydromorphes à gley (gleysols) issus de la persistance, durant la plus grande partie de l'année, d'une nappe phréatique haute reposant sur la roche mère. Ce type de sol est caractérisé par une accumulation de matière 
organique peu décomposée. En bordure de ces sols hydromorphes sont généralement observé des redoxisols argileux (Feteke et al., 2004).

\section{Caractéristiques pluviométriques de la zone d'étude}

La précipitation moyenne de ces quatre dernières années (2008-2011) est de 1726 $\mathrm{mm}$. L'année 2009 a enregistrée la plus haute valeur (1966 mm de pluie). Le mois d'août restant le plus pluvieux avec une précipitation de $369 \mathrm{~mm}$.

\section{Méthodologie \\ Dispositif de recherche}

Le dispositif expérimental utilisé est constitué de placettes semi permanentes circulaires de $20 \mathrm{~m}$ de rayon centrées sur chacun des arbres de Moabi étudiés. Il s'agit d'un dispositif de suivi de la croissance du Moabi mis en place par la Société Pallisco depuis 2008 (Doucet et al., 2012), avec 79 arbres suivis initialement (Figure 1). La présente étude a porté uniquement sur les 60 arbres régulièrement suivis entre septembre 2008 et décembre 2012.

\section{Collecte des données}

A l'intérieur de la zone de concurrence définie autour de chaque arbre central de Moabi étudié (placette semi-permanente), tous les arbres ont été identifiés (espèce et famille botanique) et mesurés (diamètre de référence et type de houppier) à l'aide d'un mètre ruban. Un clinomètre de marque «Suunto » a permis de déterminer les pentes des placettes. Une boussole de marque «Suunto » a été utilisée pour estimer les azimuts des arbres voisins à l'arbre central. Un appareil laser «Leica DISTO » a permis de déterminer les distances des arbres voisins par rapport à l'arbre central. Un GPS de marque « Garmin GPS map 62 » a été utilisé pour déterminer les positions des arbres à étudier. Des fiches de collecte de données ont permis de relever toutes ces informations.

Le statut social des arbres centraux a été évalué sur la base des codes de classification établit par Dawkins (1958) (Moravie et al., 1999). Au terme de la collecte de données, 786 arbres compétiteurs ont été identifiés dans les 60 placettes semi permanentes de Moabi.

\section{Analyse des données}

\section{Détermination des paramètres de croissance}

L'accroissement moyen annuel en diamètre $(\Delta \mathrm{d})$ de chaque arbre est calculé à partir de la formule :

$\Delta d\left(c m \cdot a n^{-1}\right)=\left(d_{n^{\prime}} /_{d_{1 n^{\prime}}}\right) / n$

L'accroissement en surface terrière $(\Delta \mathrm{g})$ de chaque arbre est calculé suivant la formule :

$\Delta g\left(m^{2} \cdot h a^{-1} \cdot a n^{-1}\right)=\pi\left(\frac{\left.d_{n}-d_{1 n \prime}\right)^{2}}{4 n}\right)$ où : $\mathbf{d}_{\mathbf{n}}=$ diamètre de l'arbre à l'année $\mathrm{n} ; \mathbf{d}_{\mathbf{1}}=$ diamètre de l'arbre à l'année $1 ; \mathbf{n}=$ nombre d'années entre deux mesures; $\mathrm{n}^{\prime}=$ indice représentant le nombre d'années entre deux mesures

Les indices de compétition déterminés portent sur deux classes distinctes: ceux dépendants de la distance et ceux qui en sont indépendants (Tableau 1). Les indices indépendants de la distance expriment la densité locale et le statut social (indice d'éclairement du houppier) des arbres par rapport au peuplement, alors que les indices dépendants de la distance expriment ces mêmes valeurs dans l'espace.

\section{Analyse des variations de la croissance}

Les accroissements en diamètre et en surface terrière en fonction des différentes variables mesurées ont été évaluées par analyses statistiques simples et multivariées à partir du logiciel STATISTICA 6.0. Les corrélations de la croissance avec les variables qualitatives de statut social (classes d'exposition) et de diamètre (classes de diamètre) des arbres, ont été déterminées par analyses de variance (ANOVA) à un facteur et ont permis d'expliquer ainsi leur effet sur la trajectoire de croissance des espèces étudiées. L'hétérogénéité des variances a été préalablement vérifiée par le Test de Brown et Forsythe d'Homogénéité des Variances. En cas de test significatif $(\mathrm{P}<0,05)$, la différence 
d'accroissement diamétrique entre les classes qualitatives est analysée ensuite par le Test HSD de Turkey afin de déceler les classes d'individus concernées.

Pour les variables quantitatives décrivant soit la morphologie des arbres (le diamètre), soit la compétition du peuplement (indices de compétition), leurs corrélations avec les variables de croissance ont été analysées par des régressions simples qui ont permis d'apprécier la relation linéaire pouvant exister entre elles et le niveau de variation qu'elles exercent sur la croissance. La performance de ces variables à expliquer les variations de la croissance a été appréciée à travers les courbes de régression et les coefficients de détermination $\left(\mathrm{R}^{2}\right)$ ainsi que de corrélation $(r)$ obtenus de ces régressions.

Pour déterminer l'effet combiné de l'ensemble des variables qualitatives et quantitatives sur la croissance des espèces, une approche d'analyse multivariée recommandée par Hill et Smith (1976) a permis de visualiser les corrélations potentielles entre variables et d'identifier les principaux facteurs de variation de la croissance inhérents au peuplement. Il s'agit d'une approche combinant les analyses en correspondance multiple (ACM) de variables qualitatives et les analyses en composantes principales (ACP) de variables quantitatives qui permet de synthétiser l'information principale (facteur de variation) véhiculée par l'ensemble des variables. L'effet combiné des variables est déterminé dans un premier temps par analyse visuelle des cercles de corrélation, et dans un second temps par analyse statistique des matrices de corrélation obtenues. Pour toute position en angle droit entre variables par rapport au centre du cercle de corrélation, la relation est jugée inexistante et donc l'effet non significatif. En cas de position autre que l'angle droit entre variables, la relation est jugée existante et sa pertinence évaluée à travers les valeurs de $r$ obtenues des matrices de corrélation.

Détermination des variables prédictives potentielles de modélisation de la croissance

La détermination des variables potentielles en vue de la modélisation de la croissance du Moabi est réalisée par analyse en régression multiple avec une démarche de recherche exhaustive et selon la méthode des moindres carrés. Elle consiste à procéder à une recherche exhaustive par itération, de combinaisons statistiquement possibles (variance résiduelle et Critère d'Information d'Akaike ou AIC minimisés) de variables explicatives (indices et paramètres de forme des arbres déterminés) permettant d'expliquer les variables de croissance. Les régressions multiples entre chacune des variables de croissance ( $\Delta \mathrm{d}$ et $\Delta \mathrm{g}$ ) et l'ensemble des variables explicatives déterminées ont été réalisées par itérations de façon à minimiser l'écart-type résiduel entre les valeurs de croissance observées et celles estimées. Le Test du $\mathrm{Chi}^{2}$ a permis de relever le degré de corrélation entre les différentes variables prédictives de l'accroissement en diamètre et en surface terrière du Moabi. En effet, cette corrélation est jugée non significative pour une valeur du Chi ${ }^{2}$ comprise entre 0 et 2,70 ; significative entre 2,70 et 3,84 et très significative lorsque supérieure à 3,84 . Les variables présentes dans les dix premières combinaisons ayant fourni les plus bas AIC ont été retenues comme potentiellement utilisables dans des modèles prédictifs de la croissance du Moabi. 


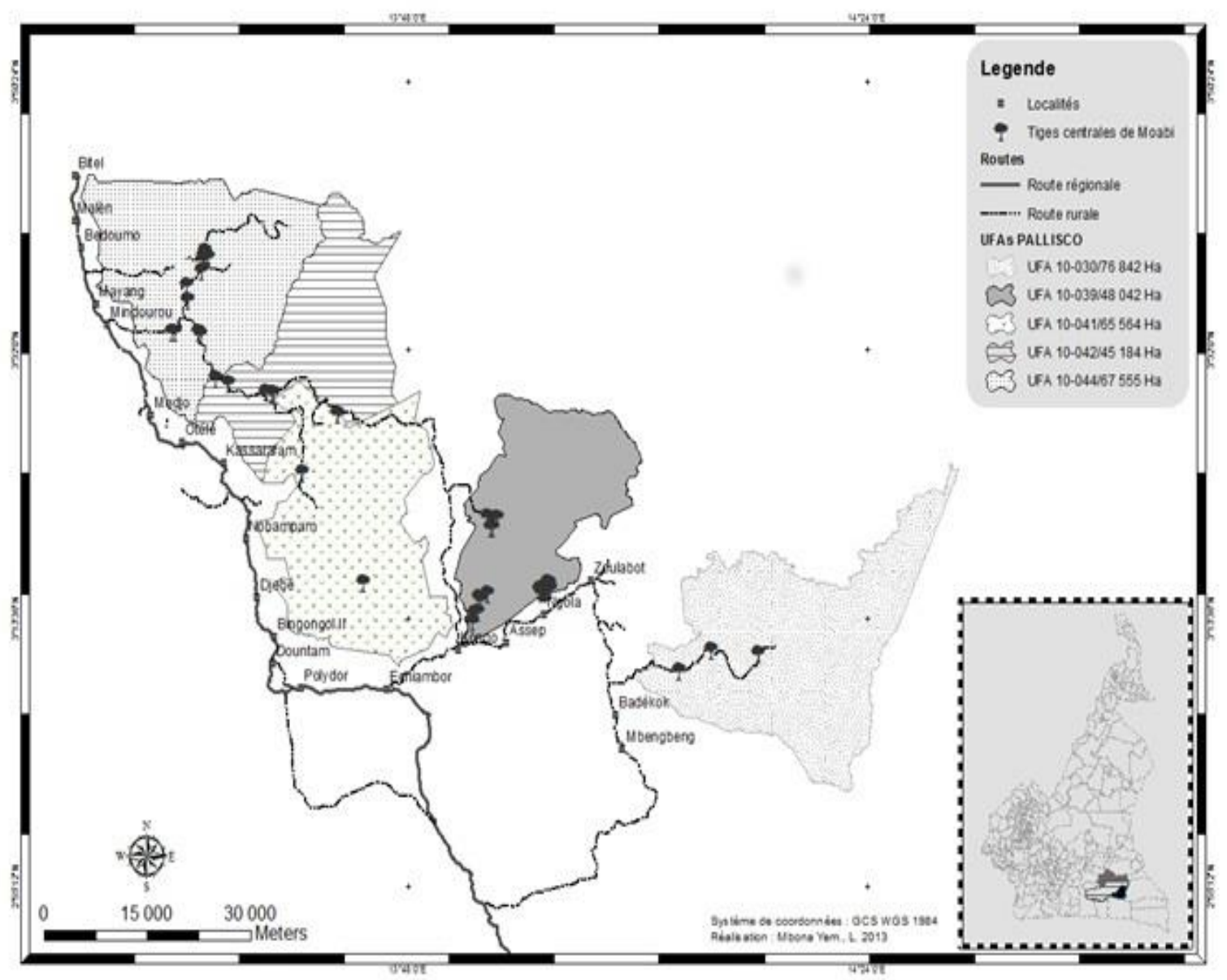

Figure 1 : Localisation des arbres centraux de Moabi des placettes circulaires semi-permanentes de $20 \mathrm{~m}$ de rayon. Atlas forestier Interactif du Cameroun version 2011.

Tableau 1 : Liste des indices de compétition déterminés pour le Moabi.

\begin{tabular}{|c|c|c|}
\hline Variables et description & $\begin{array}{c}\text { Formule de calcul } \\
\end{array}$ & Références \\
\hline $\begin{array}{l}I D \text { : indice d'exposition du houppier à la } \\
\text { lumière }\end{array}$ & $\begin{array}{c}\text { ID1 : houppier ne recevant pas de lumière } \\
\text { ID2 : houppier recevant peu de lumière } \\
\text { ID3 : houppier recevant moyennement la } \\
\text { lumière } \\
\text { ID4 : houppier recevant verticalement la lumière } \\
\text { ID5 : houppier recevant entièrement la lumière }\end{array}$ & $\begin{array}{c}\text { Dawkins } \\
\text { (1958) }\end{array}$ \\
\hline $\begin{array}{l}N V T: \text { nombre d'arbres du peuplement } \\
\text { ramené à l'hectare/nombre d'arbres } \\
\text { voisins totaux }\left(\mathrm{ha}^{-1}\right)\end{array}$ & $N V T=\sum k$ & $\begin{array}{c}\text { Steneker et } \\
\text { Jarvis (1963) }\end{array}$ \\
\hline $\begin{array}{l}N V D: \text { nombre à l'hectare des arbres du } \\
\text { peuplement de diamètre supérieur à } \\
\text { l'arbre considéré /nombre d'arbres voisins } \\
\text { imposant }\left(\text { ha }^{-1}\right)\end{array}$ & $N V D=\sum_{k=1}^{k=n_{g}} \mathrm{k}_{\mathrm{g}}$ & $\begin{array}{c}\text { Steneker et } \\
\text { Jarvis (1963) }\end{array}$ \\
\hline
\end{tabular}




\begin{tabular}{|c|c|c|}
\hline Variables et description & Formule de calcul & Références \\
\hline $\begin{array}{l}S T T: \text { surface terrière du peuplement } \\
\text { ramené à l'hectare/ Surface Terrière } \\
\text { cumulée à l'hectare de la totalité des } \\
\text { arbres voisins }\left(\mathrm{m}^{2} \text {.ha- } 1\right) /\end{array}$ & $S T T=\sum_{k=1}^{k=N V T} \frac{\pi}{4} * d h p_{k}^{2}$ & $\begin{array}{l}\text { Steneker et } \\
\text { Jarvis (1963) }\end{array}$ \\
\hline $\begin{array}{l}S T D \text { : surface terrière à l'hectare des } \\
\text { arbres du peuplement de diamètre } \\
\text { supérieur à l'arbre considéré/surface } \\
\text { terrière cumulée à l'hectare des arbres } \\
\text { voisins imposant }\left(\mathrm{m}^{2} \text {.ha-1) }\right.\end{array}$ & $S T D=\sum_{k=1}^{k=N V D} \frac{\pi}{4} * d h p_{k}^{2}$ & $\begin{array}{c}\text { Steneker et } \\
\text { Jarvis (1963) }\end{array}$ \\
\hline $\begin{array}{l}\text { SST/dist. : surface terrière du peuplement } \\
\text { pondérée par la distance avec l'arbre } \\
\text { considéré }(\mathrm{m} / \mathrm{ha})\end{array}$ & $\frac{S S T}{d i t}=\sum_{k=1}^{k=N V T} \mathrm{ST}_{\mathrm{k}} / \mathrm{dist}_{\mathrm{k}}$ & $\begin{array}{l}\text { Steneker et } \\
\text { Jarvis (1963) }\end{array}$ \\
\hline $\begin{array}{l}\text { SSTr/dist. : surface terrière relative du } \\
\text { peuplement pondérée par la distance avec } \\
\text { l'arbre considéré }\left(\mathrm{m}^{-1} 1\right)\end{array}$ & $\frac{S S T r}{d i t}=\sum_{k=1}^{k=N V T} \mathrm{ST}_{\mathrm{k}} /\left(\right.$ dist $\left._{\mathrm{k}} * \mathrm{ST}\right)$ & $\begin{array}{l}\text { Daniels et al. } \\
\quad \text { (1986) }\end{array}$ \\
\hline $\begin{array}{l}\text { SDhp/dist. : diamètre cumulé des arbres } \\
\text { du peuplement pondéré par la distance } \\
\text { avec l'arbre considéré }\left(\mathrm{m}^{-1}\right)\end{array}$ & $\frac{S D h p}{d i s t}=\sum_{k=1}^{k=N V T} \frac{1}{\text { dist }_{k}} * \frac{D H P_{k}}{D H P}$ & $\begin{array}{c}\text { Steneker et } \\
\text { Jarvis (1963) }\end{array}$ \\
\hline
\end{tabular}

\section{RESULTATS}

\section{Détermination des variables de croissance et de compétition \\ Accroissement moyen annuel}

Les résultats du calcul de l'accroissement moyen diamétrique $(\Delta \mathrm{d})$ et en surface terrière $(\Delta \mathrm{g})$ des deux espèces sur la période de 2008 à 2012 sont présentés dans le Tableau 2. L'accroissement diamétrique moyen $(\Delta \mathrm{d})$ du Moabi, pour la période de 2008 à 2012 , est estimé à $0,825 \pm 0,158 \mathrm{~cm} / \mathrm{an}$ ; Son accroissement en surface terrière $(\Delta \mathrm{g})$ est de $1,473 \pm 0,460 \mathrm{~m}^{2} / \mathrm{ha} / \mathrm{an} \times 10^{-3}$ (Tableau 2).

\section{Indice d'éclairement du houppier}

Le niveau d'éclairement du houppier des arbres est apprécié visuellement sur le terrain et ne fournit pas des informations qualitatives sur la concurrence entre arbres du peuplement pour l'accès à la lumière. Les classes définies décrivent les conditions graduelles d'exposition, allant de celles sans aucun accès possible du houppier des arbres à la lumière (classe ID1) à celles où les houppiers sont entièrement exposés à la lumière (classe ID5). Les classes d'exposition intermédiaires du houppier sont fonction de la partie (latérale ou verticale) de ce dernier la mieux exposée à la lumière (Tableau 3 ).

Indices de concurrence indépendants et dépendants de la distance

Les statistiques descriptives relatives à ces variables présentées dans le Tableau 4 indiquent non seulement les caractéristiques démographiques du peuplement, mais également illustrent la pression exercée par les arbres voisins sur les arbres centraux de Moabi étudiés. De ces statistiques, on peut constater que la densité de peuplement autour des arbres de Moabi étudiés est d'environ 113 arbres à l'hectare.

La moyenne de la plupart des variables est supérieure à 1 , traduisant ainsi la densité importante des arbres voisins et leur pression de concurrence assez forte du fait de leur position très proches des arbres centraux. Le rapport de la surface terrière du peuplement ou de la somme des diamètres des arbres concurrents par la distance qui les sépare des arbres centraux est presqu'identique et montre ainsi que pour deux peuplements de structure différente, la pression de concurrence pouvant être observée entre les arbres peut être la même. Ainsi, la pression de concurrence des 
grands arbres positionnés loin de l'arbre concurrencé équivaut à une pression exercée par de petits arbres qui en sont plus proches. Des trois indices de concurrence dépendants des distances, seul l'indice de densité locale correspondant au rapport de la somme des diamètres des arbres concurrents par la distance qui les sépare des arbres centraux (SSTr/dist) et représenté par des écarts types $(2,49)$ très élevés par rapport aux deux autres indices; ce qui traduit la grande dispersion des données. Cela prédit aussi d'une tendance instable des données en régression linéaire de l'espèce (Tableau 4).

\section{Analyse de la variation de l'accroissement annuel moyen}

Les régressions réalisées entre les valeurs de l'accroissement moyen et la taille initiale (notamment le diamètre des arbres) révèlent une corrélation linéaire significative $(p<0,05)$ (Figure 2). Ainsi donc, le diamètre initial des arbres semble être à l'origine d'une forte variation de l'accroissement en diamètre du Moabi, avec une proportion de variabilité expliquée estimée à 20,1\% (coefficient de corrélation $\left.\mathrm{R}^{2}=0,202\right)$. Pour les accroissements en surface terrière, la distribution du nuage de points de valeurs moyennes en fonction du diamètre initial des arbres montre une variation linéaire apparente et statistiquement significative (Figure 3), révélée par la droite de régression simple, le coefficient de détermination $\mathrm{R}^{2}$ de 0,272 et la probabilité $p<0,001$ obtenus.

\section{Effet des classes diamétriques des arbres sur la croissance}

Des différences de croissance entre individus de classes de diamètres différents ont été observées. En effet, l'ANOVA à un facteur réalisé entre la moyenne des accroissements annuels diamétriques pris par classe de diamètres des arbres indique des différences significatives $\left(F_{(7,52)}=8,179 ; p<\right.$ $0,001)$ avec une proportion de variabilité expliquée de $52,4 \%\left(\mathrm{R}^{2}=0,524\right)$ (Tableau 5). Le test HSD de Tuckey, qui fait une comparaison multiple entre les moyennes d'accroissement par classe de diamètres, associé à cette analyse met en exergue les différences significatives entre les petites classes $(20$ à $40 \mathrm{~cm})$ et les plus grandes classes $(40$ à $80 \mathrm{~cm}$, et 90 à $110 \mathrm{~cm}$ ). Aucune différence significative n'a été observée entre d'une part les individus des petites classes de diamètre, et d'autre part entre ceux des individus des grandes classes de diamètre. $\mathrm{La}$ plus petite croissance est enregistrée dans la classe CD8 avec la valeur moyenne de 0,255 $\pm 0,037 \mathrm{~cm} / \mathrm{an}$, et la plus grande croissance dans la classe CD9 avec la valeur moyenne de $1,321 \pm 0,658 \mathrm{~cm} / \mathrm{an}$.

L'ANOVA à un facteur réalisée entre la moyenne des accroissements moyens en surface terrière pris par classe de diamètres des arbres montre des différences significatives de croissance $\left(F_{(7,52)}=9,390 ; p\right.$ $<0,000)$, avec une proportion de variabilité expliquée de $55,83 \%\left(\mathrm{R}^{2}=0,558\right)$. Les groupes d'arbres exprimant la variabilité d'accroissement en diamètre de 52,4\% (Tableau 5) après le test HSD de Tuckey appartiennent aux classes diamétriques 20-30 $\mathrm{cm}, 30-40 \mathrm{~cm}$ et $80-90 \mathrm{~cm}$ d'une part, puis 60 $70 \mathrm{~cm}, 90-100 \mathrm{~cm}$ et $100-110 \mathrm{~cm}$ d'autre part. Aucune différence significative n'a été observée entre d'une part, les individus des petites classes de diamètre, et d'autre part, entre ceux des individus des grandes classes de diamètre. La plus petite croissance est enregistrée dans la classe CD6 avec la valeur moyenne de $0,129 \pm 0,012 \mathrm{~m}^{2} / \mathrm{ha} / \mathrm{an} \times 10^{-3}$, et la plus grande croissance dans la classe CD5 avec la valeur moyenne de 4,491 $\pm 1,039$ $\mathrm{m}^{2} / \mathrm{ha} / \mathrm{an} \times 10^{-3}$.

\section{Effet de l'indice d'éclairement du houppier sur la croissance}

L'évaluation de l'influence du statut social sur la croissance des arbres de Moabi étudiés par ANOVA à un facteur réalisée, entre les moyennes d'accroissement diamétrique et les classes de statut social (indice de Dawkins), a révélé un effet significatif $\left(F_{(4,55)}=3,764 ; p=0,0089\right)$ avec une variance totale de l'accroissement expliquée par cet indice de compétition 
estimée à $21,5 \%\left(\mathrm{R}^{2}=0,2149\right)$. La plus petite valeur d'accroissement moyen en diamètre $(0,38 \pm 0,42 \mathrm{~cm} / \mathrm{an})$ est observée dans la classe ID1, arbres dont les cimes sont entièrement ombragées, donc qui ne reçoivent pas de lumière. Par contre, la valeur d'accroissement diamétrique moyen la plus importante $(1,12 \pm 0,357 \mathrm{~cm} / \mathrm{an}) \quad$ est enregistrée dans la classe ID4 des arbres entièrement exposés à la lumière verticalement et ombragés latéralement (Tableau 6).

\section{Effet des indices de compétition indépendants et dépendants de la distance sur la croissance}

Les Figures 4 et 5 permettent d'apprécier le degré de liaison entre les variables dépendantes (accroissement diamétrique moyen et en surface terrière moyenne) et les principales variables explicatives (Indices de Densité Locale) relatives au peuplement de Moabi. En effet, il ressort de la Figure 4 que seul l'indice de densité locale NVD (nombre de voisins imposants par rapport au diamètre de l'arbre central) explique le mieux l'accroissement en diamètre $\left(R^{2}=33,10\right)$ et l'accroissement en surface terrière $\left(R^{2}=30,8\right)$. D'autre part, l'indice de densité locale STD (somme de surface terrière voisins imposants par rapport au diamètre de l'arbre central) a également un taux appréciable de l'accroissement en diamètre $\left(R^{2}=21,70\right)$ et l'accroissement en surface terrière $\left(\mathrm{R}^{2}=23,01\right)$ (Figure 5$)$. En outre, les indices de concurrence NVT et SST avec une corrélation comprise entre 0,001 et 0,059 n'explique que très faiblement la variabilité de la croissance en diamètre et en surface terrière du Moabi.

Les Figures 6 et 7 présentent les distributions d'accroissement moyen diamétrique et en surface terrière du Moabi en fonction des indices de concurrence SSTr/dist (Figure 6), SST/dist (Figure 7). Seul l'indice de concurrence STTr/Dist est la variable qui explique le mieux l'accroissement en diamètre $\left(\mathrm{R}^{2}=25,50\right)$ et l'accroissement en surface terrière $\left(R^{2}=20,00\right)$ de manière soutenue. En outre, l'indice de concurrence SDHP/dist montre une très faible capacité d'explication de la variabilité de croissance en diamètre et en surface terrière du Moabi avec des coefficients de corrélations inférieure à 0,006 .

\section{Principaux facteurs de variation de la croissance}

Les corrélations entre variables explicatives de compétition de croissance sont visualisées dans le cercle de corrélation obtenu des analyses multivariées mixtes (Figure 8). Les analyses multivariées mixtes réalisées pour les variables qualitatives (classe de statut social) et quantitatives (indices de compétition et accroissement en diamètre des arbres et diamètre des arbres) auxquelles les variables de croissance du Moabi ont été associées, révèlent l'existence de deux principaux facteurs expliquant l'inertie totale portée par les variables analysées. Le cercle de corrélation décrit par ces deux principaux facteurs répartit les variables en trois principaux groupes :

(1) Les variables ID (niveau d'éclairement du houppier) et $\mathrm{D}$ (diamètre des arbres) caractérisant le statut social des arbres centraux étudiés, ainsi que les variables de croissance $(\Delta \mathrm{d}$ et $\Delta \mathrm{g})$;

(2) Les variables NVT (densité totale), STT (surface terrière totale), SST/dist (surface terrière totale relative à la distance) et SDHP/dist (somme des diamètres relative à la distance) caractérisant la densité du peuplement ;

(3) Les variables NVD (densité des concurrents imposants), STD (surface terrière des concurrents imposants et SSTr/dist (surface terrière relative par rapport à la distance) caractérisant le statut social du peuplement.

Dans ce cercle de corrélation, $78,46 \%$ de la variance est expliquée par les deux axes représentés. L'axe 1 représentant le facteur d'inertie Fact. 1 expliquant la plus grande proportion $(62,04 \%)$ de l'inertie totale véhiculée par les différentes variables explicatives, oppose les variables décrivant le statut social du peuplement à celles décrivant 
la densité locale du peuplement, tandis que l'axe 2 représentant le facteur Fact. 2 (16,42\% de l'inertie expliquée) oppose les variables décrivant le statut social des arbres à celles décrivant la densité locale du peuplement. La disposition des variables qualitatives et quantitatives de croissance dans le cercle de corrélation montre une forte corrélation d'une part entre les variables du statut social du peuplement, d'autre part entre les variables de densité locale du peuplement, et enfin entre les variables du statut social des arbres. D, ID, NVD, NVT, STT sont les variables les plus proche du cercle de corrélation, donc explique le mieux la variabilité de la croissance du Moabi. En outre, ces corrélations sont perceptibles à travers le Tableau 7 de corrélation des variables et des principaux facteurs de variation de la croissance obtenus des analyses multivariées.

\section{Variables prédictives potentielles de la croissance du Moabi}

Les variables prédictives de l'accroissement annuel moyen sont celles susceptible d'être utilisées dans des modèles de prédiction de la croissance des espèces. Les analyses en régression multiple réalisée par recherche exhaustive de variables pertinentes avec la méthode des moindres carrés ont fourni une série de combinaisons statistiques pertinentes de variables permettant d'expliquer le maximum de variabilité de la croissance. Les dix premières combinaisons ayant le plus bas critère d'information d'Akaike (AIC) et donc les plus pertinentes de la série sont présentées dans les Tableaux 7 et 8. Le seul trait morphologique des arbres qui s'est révélé pertinent dans la prédiction de la croissance du Moabi est le diamètre (D). Cette variable interviendrait particulièrement dans la prédiction de l'accroissement en diamètre et en surface terrière du Moabi. A partir du Test de $\mathrm{Chi}^{2}$ qui mesure le degré de corrélation entre les variables prédictives, on n'en déduit que toutes les combinaisons enregistrées dont les variables prédictives de l'accroissement en diamètre et en surface terrière ayant une valeur du $\mathrm{Chi}^{2}$ supérieure à 3,84 sont considérées comme étant très corrélées. Les meilleures combinaisons de variables pertinentes sont les premières de chaque série figurant dans les Tableaux 8 et 9 . La combinaison prédisant le mieux l'accroissement diamétrique du Moabi comporte trois variables (deux indices indépendants des distances (NVD, NVT) et un indice dépendant des distances (SDHP/dist)) avec un AIC de 83,51.

Tableau 2 : Statistiques descriptives des données d'accroissement en diamètre $(\Delta \mathrm{d})$ et en surface terrière $(\Delta \mathrm{g})$ du Moabi.

\begin{tabular}{lcc}
\hline Fonctions statistiques & $\boldsymbol{\Delta d} \mathbf{( C m} / \mathbf{a n})$ & $\Delta \mathbf{g}\left(\mathbf{m}^{\mathbf{2}} / \mathbf{h a} / \mathbf{a n} \mathbf{x} \mathbf{~ 1 0}^{-\mathbf{3}}\right)$ \\
\hline Moyenne & 0,825 & 1,473 \\
Intervalle de confiance & 0,158 & 0,460 \\
Erreur-type & 0,081 & 0,234 \\
Écart-type & 0,625 & 1,818 \\
Variance de l'échantillon & 0,391 & 3,306 \\
Nombre d'arbres & & \multirow{2}{*}{60} \\
\hline
\end{tabular}


Tableau 3: Distribution des effectifs d'arbres centraux de Moabi en fonction des classes diamétriques (CD) et du statut social (ID).

\begin{tabular}{ccccccccccccc}
\hline \multirow{2}{*}{$\begin{array}{c}\text { Classe de } \\
\text { statut (ID) }\end{array}$} & \multicolumn{1}{c}{ Classes de diamètres (CD en cm) } & \multicolumn{3}{c}{ Total } \\
\cline { 2 - 12 } & CD 20 & CD 30 & CD 40 & CD 50 & CD 60 & CD 70 & CD 80 & CD 90 & $\begin{array}{c}\text { CD } \\
\text { 100 }\end{array}$ & $\begin{array}{c}\text { CD } \\
\mathbf{1 1 0}\end{array}$ \\
\hline $\mathrm{ID}_{1}$ & 1 & 1 & 0 & 0 & 0 & 0 & 0 & 0 & 0 & 0 & 2 \\
$\mathrm{ID}_{2}$ & 10 & 5 & 2 & 0 & 0 & 0 & 0 & 0 & 0 & 0 & 17 \\
$\mathrm{ID}_{3}$ & 1 & 4 & 2 & 0 & 0 & 0 & 1 & 0 & 0 & 0 & 8 \\
$\mathrm{ID}_{4}$ & 4 & 2 & 3 & 0 & 1 & 3 & 1 & 3 & 1 & 0 & 18 \\
$\mathrm{ID}_{5}$ & 1 & 2 & 4 & 1 & 1 & 0 & 1 & 3 & 2 & 0 & 15 \\
\hline Total & $\mathbf{1 7}$ & $\mathbf{1 4}$ & $\mathbf{1 1}$ & $\mathbf{1}$ & $\mathbf{2}$ & $\mathbf{3}$ & $\mathbf{3}$ & $\mathbf{6}$ & $\mathbf{3}$ & $\mathbf{0}$ & $\mathbf{6 0}$
\end{tabular}

ID: Statut social

CD: Classes de diametre

Tableau 4 : Statistiques descriptives des indices de concurrence indépendants et dépendants de la distance déterminés pour le Moabi.

\begin{tabular}{|c|c|c|c|c|c|c|c|}
\hline $\begin{array}{l}\text { Paramètres } \\
\text { statistiques }\end{array}$ & $\begin{array}{c}\text { NVT } \\
\text { (arbres/ha) }\end{array}$ & $\begin{array}{c}\text { NVD } \\
\text { (arbres/ha) }\end{array}$ & $\begin{array}{c}\text { STT } \\
\left(\mathrm{m}^{2} / \mathrm{ha} \times 10^{-3}\right)\end{array}$ & $\begin{array}{c}\text { STD } \\
\left(\mathrm{m}^{2} / \mathrm{ha} \times 10^{-3}\right)\end{array}$ & $\begin{array}{l}\text { SST/dist } \\
\text { (m/ha) }\end{array}$ & $\begin{array}{l}\text { SSTr/dist } \\
\left(\mathrm{m}^{-1}\right)\end{array}$ & $\begin{array}{c}\text { SDHP/dist } \\
\left(\mathrm{m}^{-1}\right)\end{array}$ \\
\hline $\begin{array}{l}\text { Moyenne/ } \\
\text { Medium }\end{array}$ & 120,88 & 46,31 & 138,32 & 87,92 & 1,81 & 2,34 & 1,70 \\
\hline $\begin{array}{l}\text { Erreur-type/Standard } \\
\text { error }\end{array}$ & 6,68 & 5,50 & 8,14 & 9,04 & 0,12 & 0,33 & 0,15 \\
\hline $\begin{array}{l}\text { Ecart-type/Standard } \\
\text { deviation }\end{array}$ & 51,73 & 42,62 & 63,04 & 70,01 & 0,94 & 2,49 & 1,16 \\
\hline Variance & 2676,33 & 1816,80 & 3973,99 & 4900,79 & 0,89 & 6,22 & 1,36 \\
\hline Minimum & 15,92 & 0 & 9,95 & 0 & 0,10 & 0,03 & 0,09 \\
\hline Maximum & 270,7 & 159,24 & 277,47 & 239,02 & 4,66 & 11,03 & 4,66 \\
\hline Nombre d'arbres & & & 60 & & & 60 & \\
\hline
\end{tabular}

N.B.: Les formules utilisées sont référencées dans le Tableau 1. 


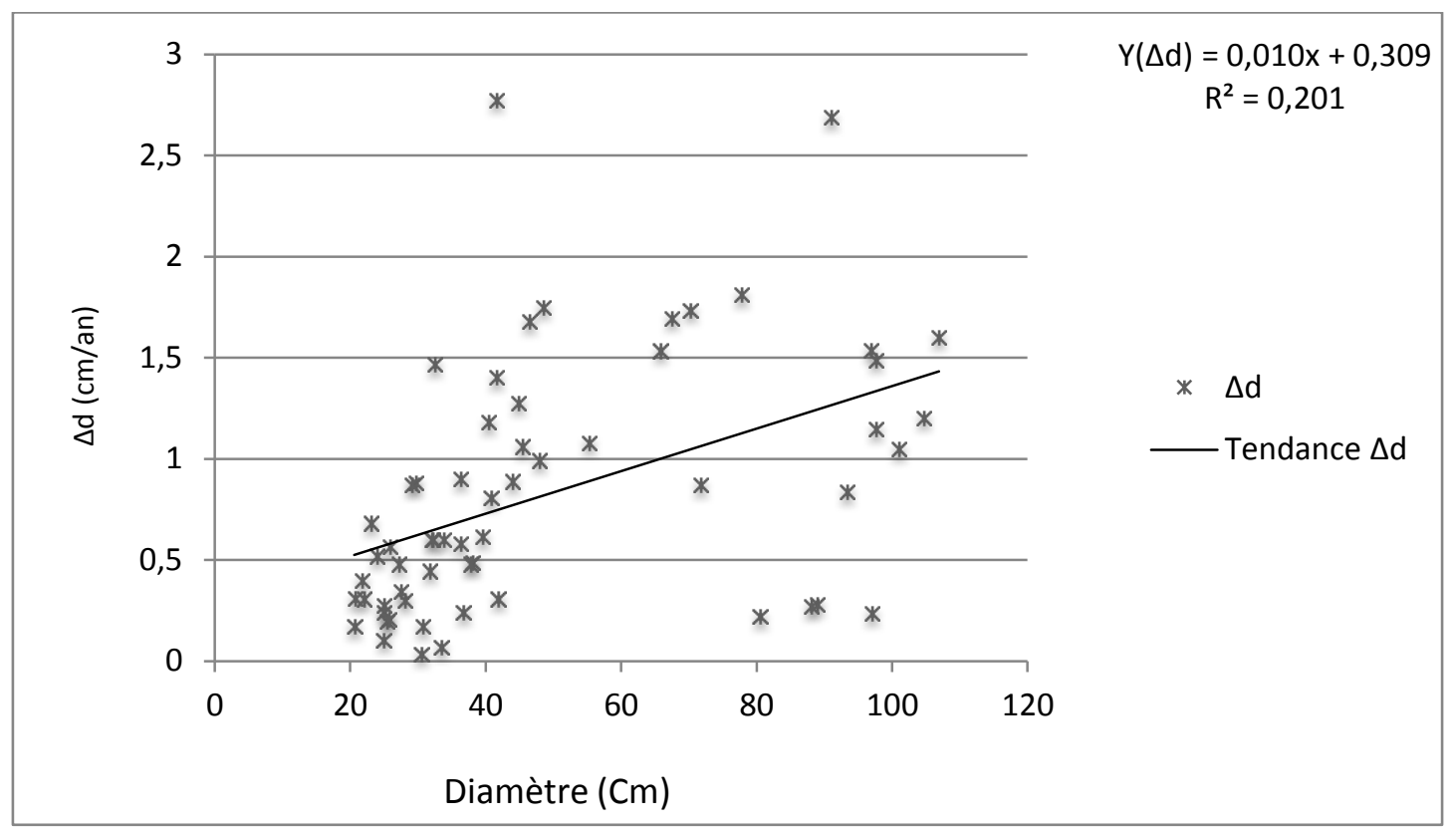

Figure 2 : Distribution des accroissements moyens diamétriques en fonction des diamètres initiaux des arbres de Moabi.

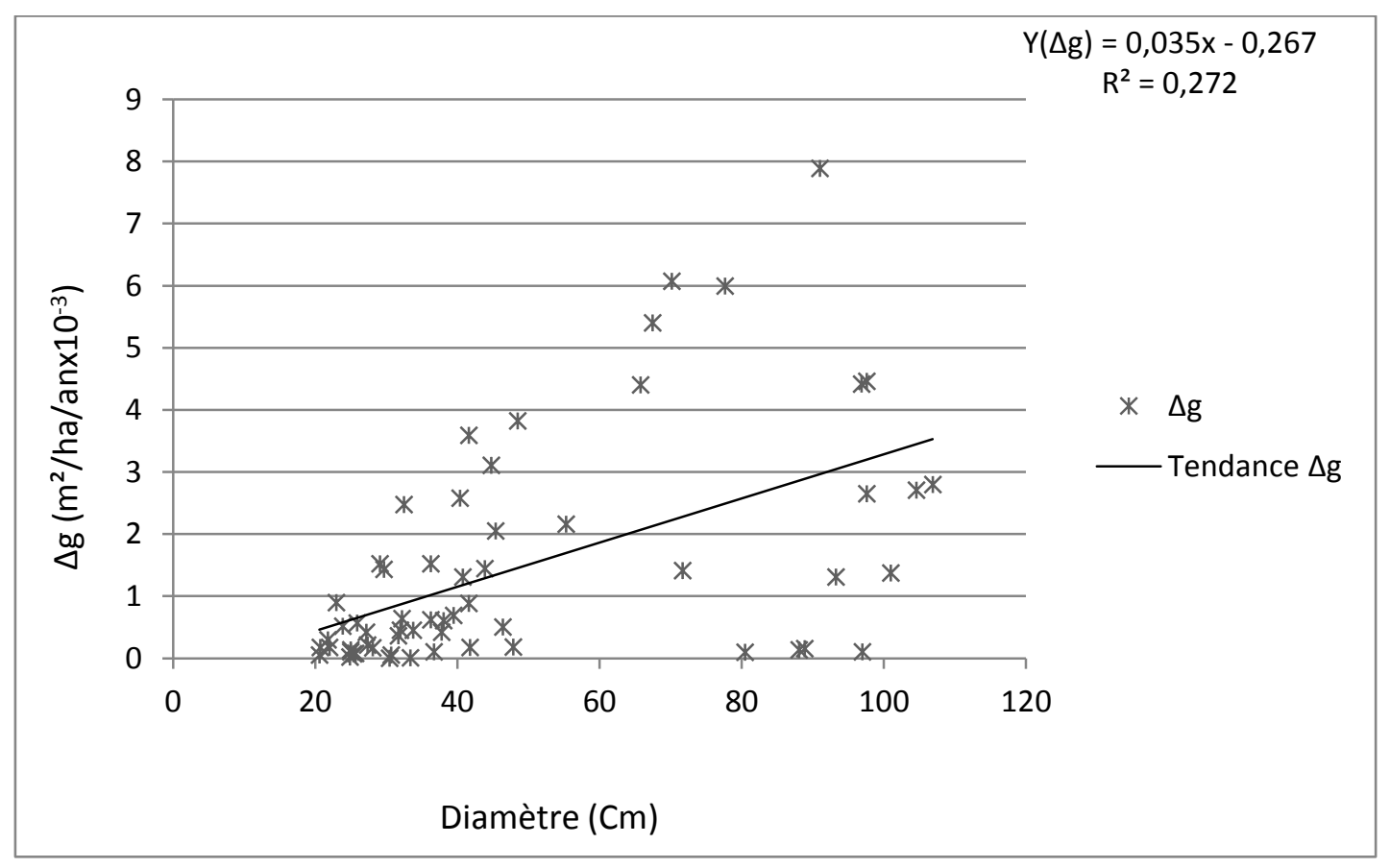

Figure 3 : Distribution des accroissements moyens en surface terrière en fonction des diamètres initiaux des arbres de Moabi. 
Tableau 1 : Accroissement moyen diamétrique $(\Delta \mathrm{d}$ en $\mathrm{cm} / \mathrm{an})$ et en surface terrière $\left(\Delta \mathrm{g}\right.$ en $\mathrm{m}^{2} / \mathrm{ha} / \mathrm{an}$ x $10^{-3}$ ) du Moabi par classe de diamètre mesuré entre septembre 2008 et décembre 2012.

\begin{tabular}{|c|c|c|c|c|c|c|c|c|c|c|c|c|}
\hline \multirow{2}{*}{\multicolumn{2}{|c|}{ Classe Ø }} & \multicolumn{6}{|c|}{$\begin{array}{l}\text { Accroissement diamétrique } \\
(\mathbf{c m} / \mathbf{a n})\end{array}$} & \multicolumn{5}{|c|}{$\begin{array}{l}\text { Accroissement surface terrière } \\
\left(\mathbf{m}^{2} / \mathrm{ha} / \text { an } \times 10^{-3}\right)\end{array}$} \\
\hline & & $\mathbf{N}$ & $\Delta d$ & $\operatorname{Max} \Delta d$ & $\operatorname{Min} \Delta d$ & Var & IC & $\Delta \mathrm{g}$ & $\operatorname{Max} \Delta \mathrm{g}$ & $\operatorname{Min} \Delta \mathrm{g}$ & Var & IC \\
\hline CD1 & {$[20-30[$} & 17 & $0,402_{b}$ & 0,880 & 0,102 & 0,054 & 0,038 & $0,402_{\mathrm{b}}$ & 1,523 & 0,02 & 0,215 & 0,076 \\
\hline $\mathrm{CD} 2$ & {$[30-40[$} & 14 & $0,520_{\mathrm{b}}$ & 1,468 & 0,034 & 0,133 & 0,066 & $0,600_{\mathrm{b}}$ & 2,476 & 0,002 & 0,443 & 0,120 \\
\hline CD3 & {$[40-50[$} & 11 & $1,282 \mathrm{a}$ & 2,772 & 0,307 & 0,409 & 0,130 & $1,786_{\mathrm{a}}$ & 3,825 & 0,173 & 1,784 & 0,272 \\
\hline CD4 & {$[60-70[$} & 3 & $1,435_{\mathrm{a}}$ & 1,694 & 1,078 & 0,102 & 0,124 & $3,987 \mathrm{a}$ & 5,399 & 2,157 & 2,758 & 0,647 \\
\hline CD5 & [70 - 80[ & 3 & $1,471_{\mathrm{a}}$ & 1,810 & 0,868 & 0,273 & 0,204 & $4,491_{\mathrm{a}}$ & 6,068 & 1,411 & 7,116 & 1,039 \\
\hline CD6 & [80 - 90[ & 3 & $0,255_{\mathrm{b}}$ & 0,279 & 0,218 & 0,001 & 0,013 & $0,129_{b}$ & 0,157 & 0,095 & 0,001 & 0,012 \\
\hline CD7 & {$[90-100[$} & 6 & $1,321_{\mathrm{a}}$ & 2,686 & 0,234 & 0,677 & 0,227 & $3,471_{\mathrm{a}}$ & 7,889 & 0,102 & 7,621 & 0,760 \\
\hline $\begin{array}{l}\text { CD8 } \\
\text { Moy }\end{array}$ & $\begin{array}{r}{[100-110[} \\
\text { nne/Medium }\end{array}$ & $\begin{array}{c}3 \\
60\end{array}$ & $\begin{array}{l}1,283_{\mathrm{a}} \\
0,825\end{array}$ & 1,600 & 1,048 & 0,081 & 0,111 & $\begin{array}{c}2,293_{\mathrm{a}} \\
1,473\end{array}$ & 2,8 & 1,375 & 0,635 & 0,310 \\
\hline
\end{tabular}

Probabilité/Probabil

ity

$\mathrm{p}<0,001 * * *$

$\mathrm{p}<0,000 * * *$

$N B$ : Seuil de signification $=0,05 ; a, b$ représentent les différences significatives qui existent entre les classes de diamètres. Lorsque les lettres sont différentes dans les classes de diamètres, c'est qu'il existe une différence significative entre les valeurs. Lorsque les mêmes lettres se retrouvent dans des classes différentes, c'est qu'il n'existe pas de différence significative entre ces valeurs, $P<0,05$ signifie que le test est significatif, ID : Statut social, CD : Classes de diamètre

Tableau 6 : Accroissement moyen en diamètre du Moabi en fonction du statut social des arbres.

\begin{tabular}{|c|c|c|c|c|c|c|c|}
\hline \multirow[t]{2}{*}{ Classe du houppier } & \multirow{2}{*}{$\begin{array}{l}\text { Nombre } \\
\text { d'arbres }\end{array}$} & \multicolumn{3}{|c|}{$\begin{array}{l}\text { Accr. en diamètre } \\
(\mathbf{c m} / \mathrm{an})\end{array}$} & \multicolumn{3}{|c|}{$\begin{array}{c}\text { Accr. en surface } \\
\text { terrière }\left(\mathrm{m}^{2} / \mathrm{ha} / \mathbf{a n} \times \mathbf{x}^{-3}\right)\end{array}$} \\
\hline & & Moy. & ET. & IC & Moy. & ET. & IC \\
\hline $\mathrm{ID}_{1}$ & 2 & 0,38 & 0,30 & 0,42 & 0,25 & 0,28 & 0,38 \\
\hline $\mathrm{ID}_{2}$ & 17 & 0,50 & 0,43 & 0,20 & 0,70 & 1,01 & 0,48 \\
\hline $\mathrm{ID}_{3}$ & 8 & 0,56 & 0,50 & 0,35 & 0,76 & 1,26 & 0,87 \\
\hline $\mathrm{ID}_{4}$ & 18 & 1,11 & 0,77 & 0,35 & 2,23 & 2,40 & 1,10 \\
\hline $\mathrm{ID}_{5}$ & 15 & 1,04 & 0,47 & 0,23 & 1,98 & 1,64 & 0,83 \\
\hline Moyenne & & 0,72 & 0,49 & 0,12 & 1,18 & 1,32 & 0,33 \\
\hline
\end{tabular}




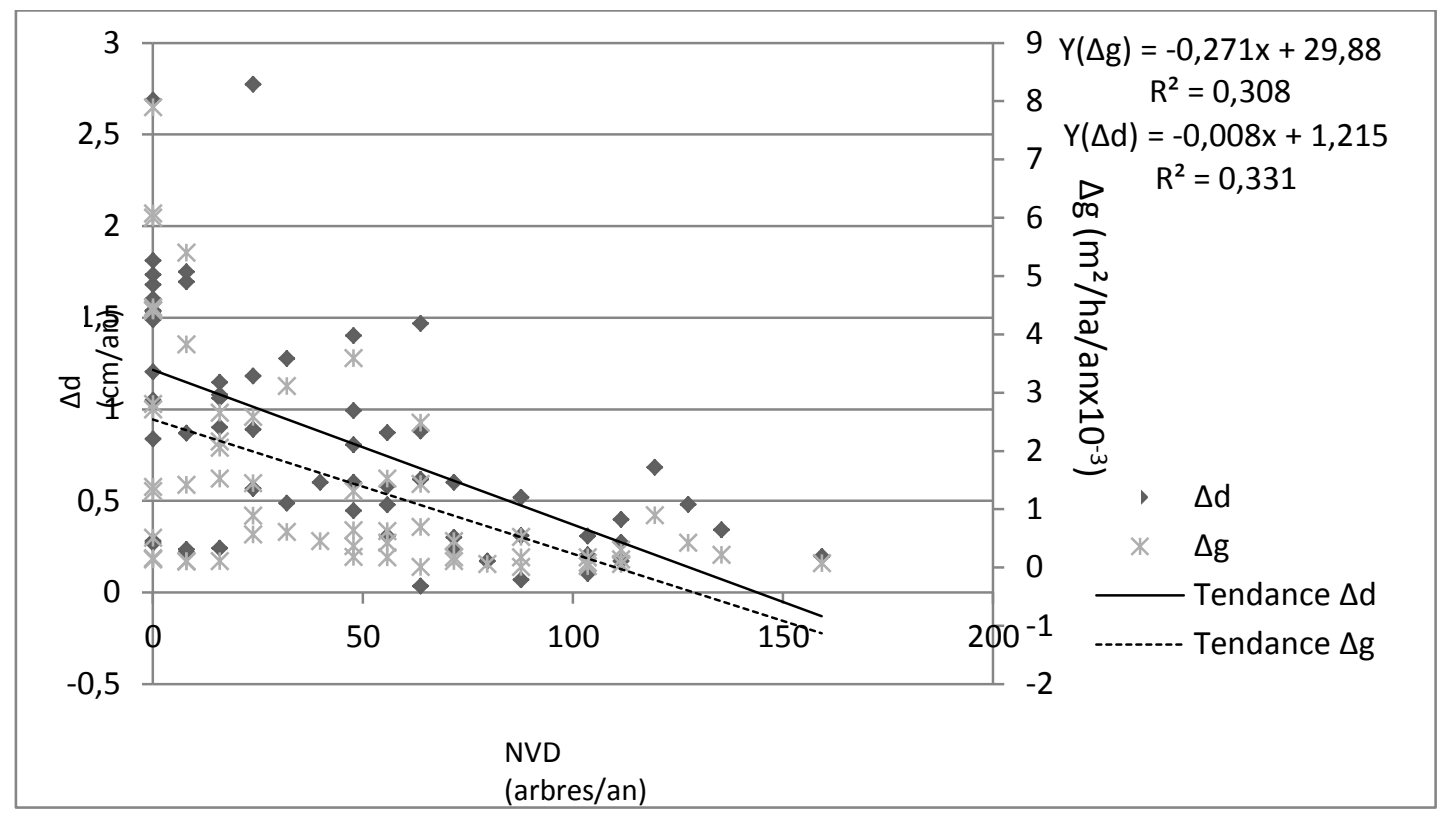

Figure 4: Distribution des accroissements annuel moyen en diamètre $(\Delta d)$ et en surface terrière $(\Delta g)$ en fonction de l'indice de concurrence indépendant de la distance NVD.

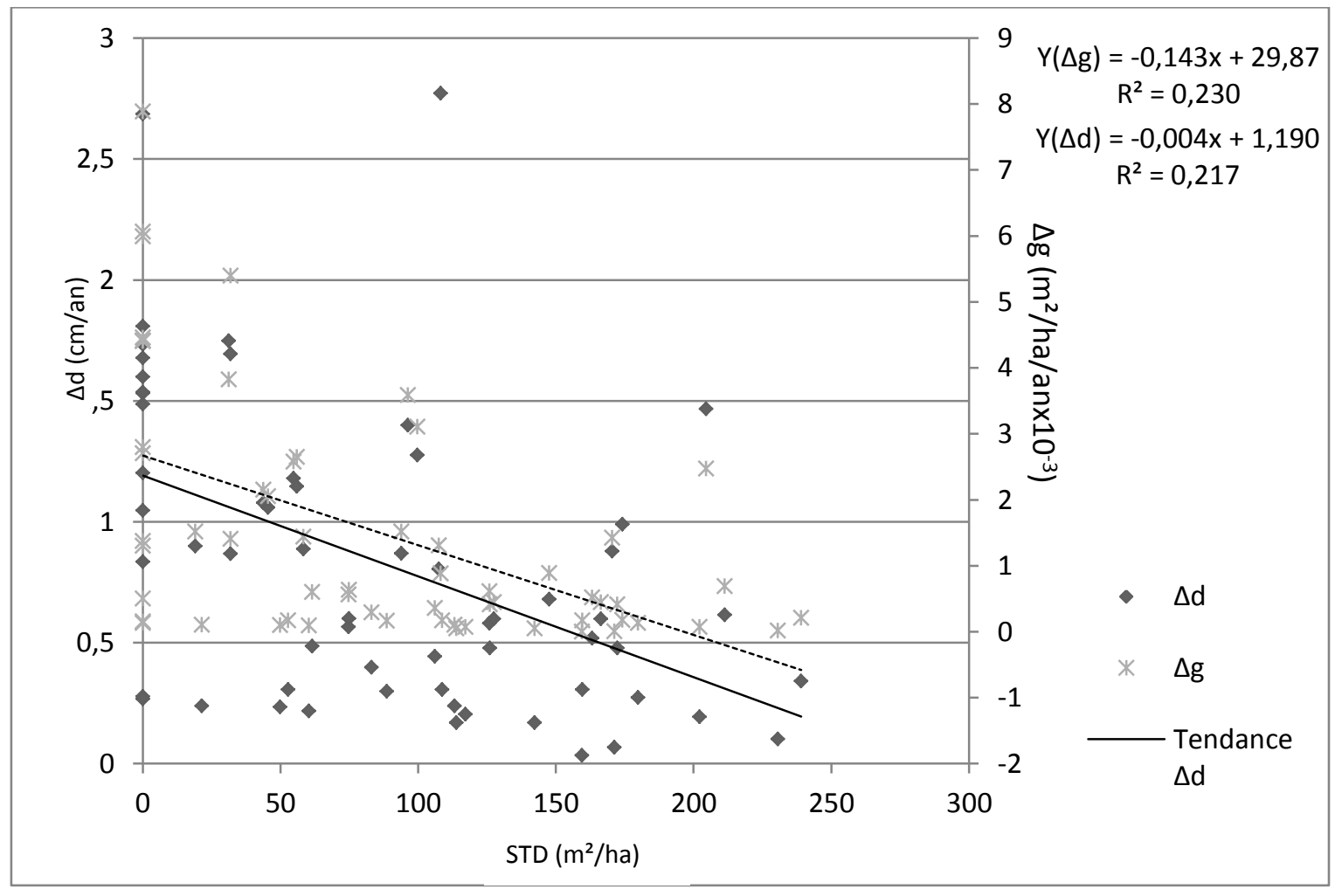

Figure 5 : Distribution des accroissements annuel moyen en diamètre $(\Delta \mathrm{d})$ et en surface terrière $(\Delta \mathrm{g})$ en fonction de l'indice de concurrence indépendant de la distance STD. 


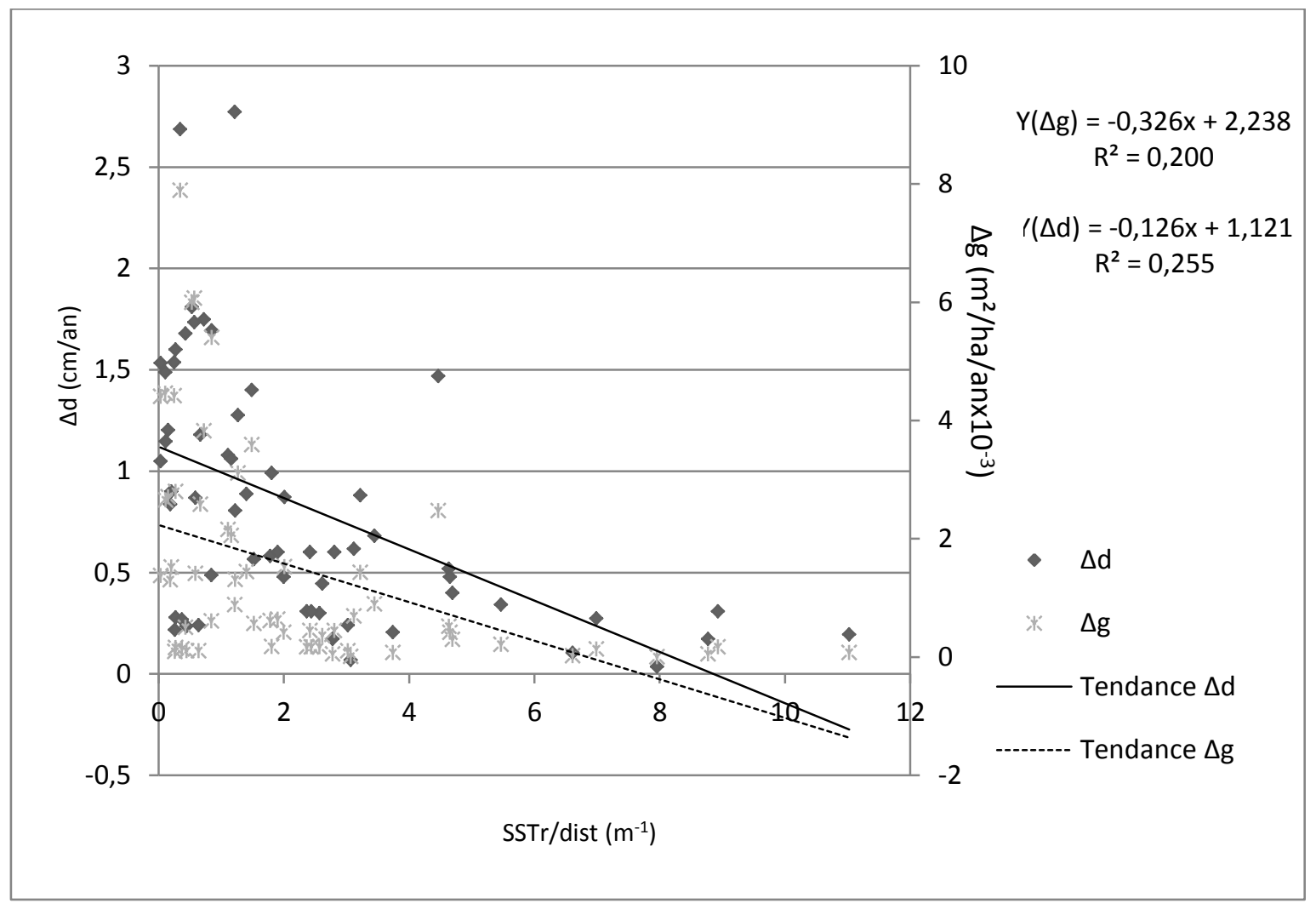

Figure 6 : Distribution des accroissements annuel moyen en diamètre $(\Delta d)$ et en surface terrière en fonction de l'indice de concurrence dépendant de la distance SSTr/dist.

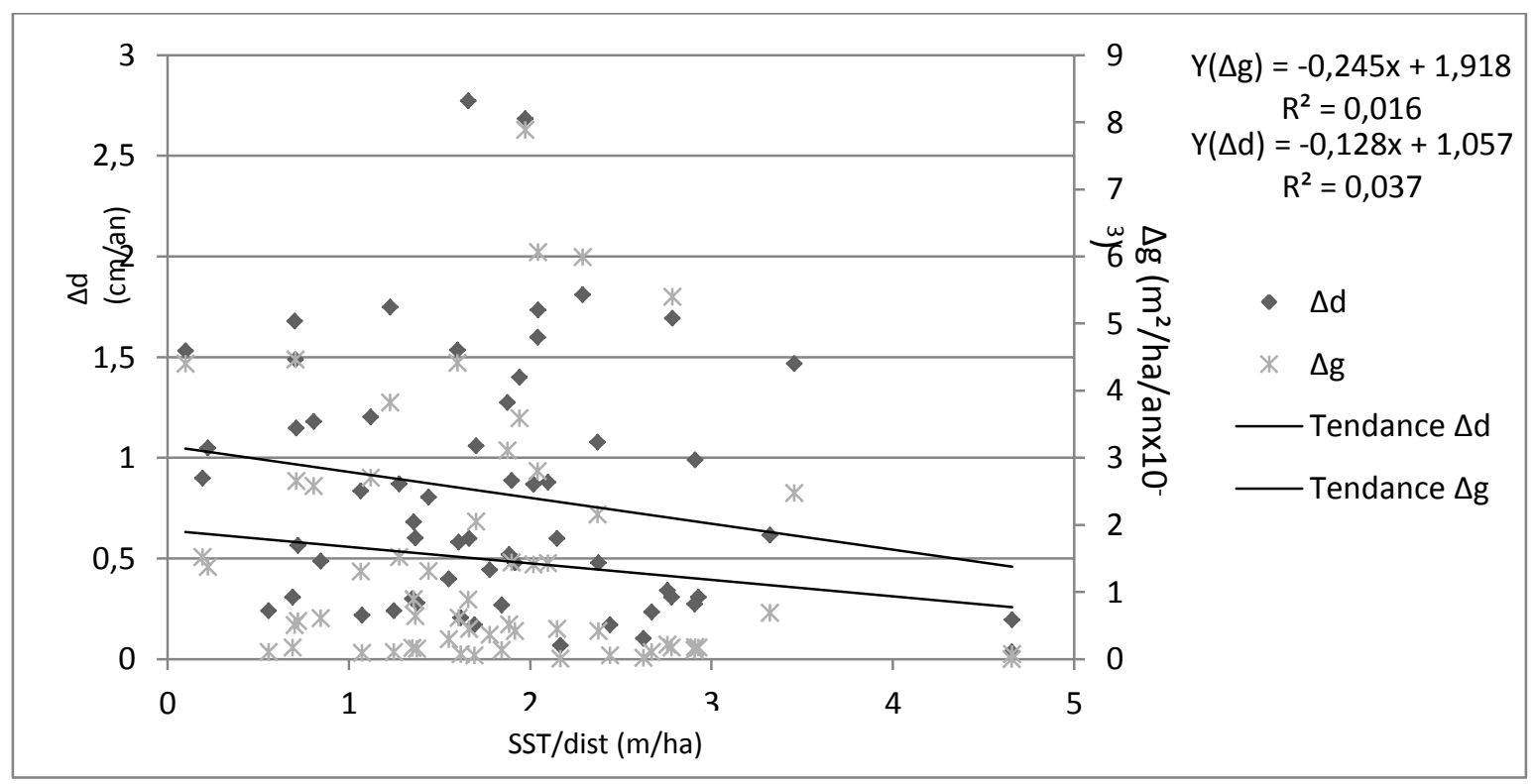

Figure 7 : Distribution des accroissements annuel moyen en diamètre $(\Delta d)$ et en surface terrière en fonction de l'indice de concurrence dépendant de la distance SST/dist. 


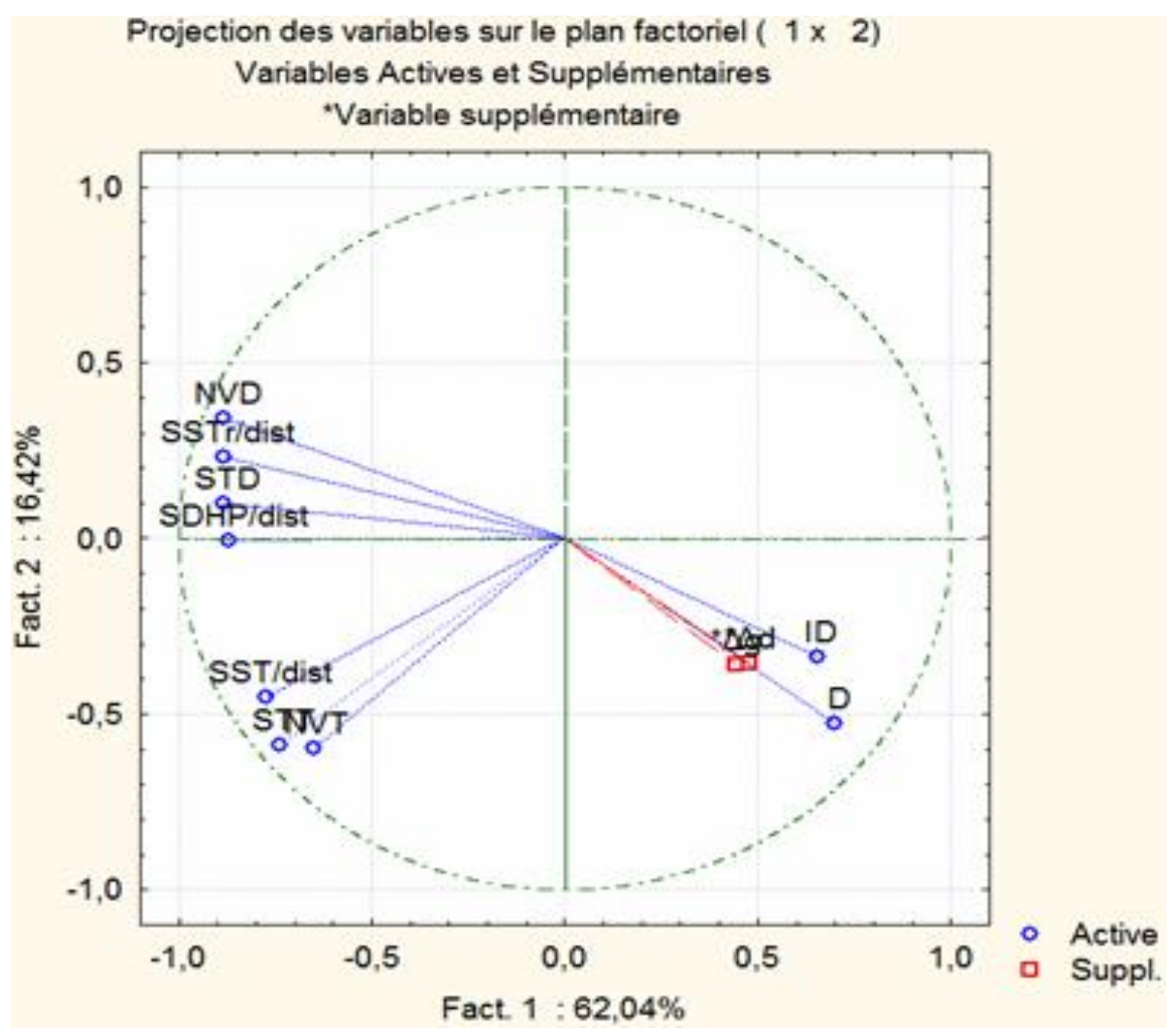

Figure 8: Représentation du cercle de corrélation des variables explicatives qualitatives et quantitatives du Moabi obtenues des analyses multivariées.

Tableau 7 : Table de corrélation des variables et des principaux facteurs (F1 et F2) de variation de la croissance obtenus des analyses multivariées.

\begin{tabular}{lccc}
\hline $\begin{array}{l}\text { Groupes de variables/Facteurs de } \\
\text { variation de l'accroissement }\end{array}$ & Variables & Fact. $\mathbf{~}$ & Fact. 2 \\
\hline Statut social & D & $\mathbf{0 , 7 0}$ & $-0,53$ \\
des arbres centraux & ID & $\mathbf{0 , 6 6}$ & $-0,34$ \\
\hline Statut social & STD & $\mathbf{- 0 , 8 8}$ & 0,09 \\
du peuplement & SSTr/dist & $\mathbf{- 0 , 8 8}$ & 0,23 \\
& NVD & $\mathbf{- 0 , 8 8}$ & 0,34 \\
\hline Densité locale & STT & $\mathbf{- 0 , 7 4}$ & $-0,59$ \\
du peuplement & SDHP/dist & $\mathbf{- 0 , 8 7}$ & $-0,01$ \\
& SST/dist & $\mathbf{- 0 , 7 7}$ & $-0,45$ \\
& NVT & $\mathbf{- 0 , 6 5}$ & $-0,60$ \\
\hline Croissance des & $\Delta \mathrm{d}$ & 0,47 & $-0,36$ \\
arbres centraux & $\Delta \mathrm{g}$ & 0,44 & 0,36 \\
\hline
\end{tabular}

N.B. : Les formules utilisées sont référencées dans le Tableau 1. 
MBONAYEM LIBOUM et K.S. BOBO / Int. J. Biol. Chem. Sci. 11(6): 2671-2692, 2017

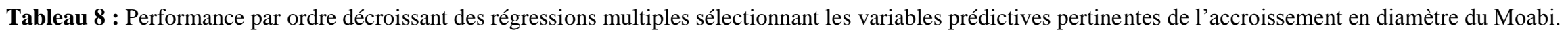

\begin{tabular}{|c|c|c|c|c|c|c|c|c|c|c|}
\hline Combinaison/Modèle & Var. 1 & Var. 2 & Var. 3 & Var. 4 & Var. 5 & Var. 6 & Degré de Liberté & AIC & Ratio V Chi ${ }^{2}$ & $\mathbf{p}$ \\
\hline 1 & NVT & NVD & SDHP/dist & & & & 3 & 83,51 & 37,41 & $3,76 \mathrm{E}-08$ \\
\hline 2 & $\mathrm{D}$ & NVT & NVD & SDHP/dist & & & 4 & 83,92 & 39,00 & $6,96 \mathrm{E}-08$ \\
\hline 3 & NVT & NVD & STD & SDHP/dist & & & 4 & 83,93 & 38,99 & $6,99 \mathrm{E}-08$ \\
\hline 4 & $\mathrm{D}$ & NVT & NVD & STD & SDHP/dist & & 5 & 84,05 & 40,87 & 9,95E-08 \\
\hline 5 & $\mathrm{D}$ & NVT & NVD & SST/dist & SDHP/dist & & 5 & 84,77 & 40,15 & $1,39 \mathrm{E}-07$ \\
\hline 6 & NVT & STT & NVD & STD & SDHP/dist & & 5 & 84,88 & 40,04 & $1,47 \mathrm{E}-07$ \\
\hline 7 & NVT & NVD & SST/dist & SDHP & & & 4 & 85,38 & 37,54 & $1,40 \mathrm{E}-07$ \\
\hline 8 & NVT & NVD & SSTr & SDHP & & & 4 & 85,43 & 37,49 & $1,43 \mathrm{E}-07$ \\
\hline 9 & NVT & STT & NVD & SDHP & & & 4 & 85,48 & 37,44 & $1,46 \mathrm{E}-07$ \\
\hline 10 & D & NVT & NVD & STD & SST/dist & SDHP/dist & 6 & 85,72 & 41,20 & $2,64 \mathrm{E}-07$ \\
\hline
\end{tabular}

Tableau 9 : Performance des régressions multiples sélectionnant les variables prédictives pertinentes de l'accroissement en surface terrière du Moabi.

\begin{tabular}{|c|c|c|c|c|c|c|c|c|c|c|}
\hline Combinaisons/modèles & Var. 1 & Var. 2 & Var. 3 & Var. 4 & Var. 5 & Var. 6 & Degré de Liberté & AIC & Ratio V Chi ${ }^{2}$ & $\mathbf{p}$ \\
\hline 1 & $\mathrm{D}$ & NVT & NVD & STD & SST/dist & SDHP/dist & 6 & 503,49 & 44,10 & $7,07 \mathrm{E}-08$ \\
\hline 2 & $\mathrm{D}$ & NVT & NVD & STD & SDHP & & 5 & 503,79 & 41,79 & $6,49 \mathrm{E}-08$ \\
\hline 3 & $\mathrm{D}$ & STT & NVD & STD & SST/dist & SDHP/dist & 6 & 504,27 & 43,32 & $1,01 \mathrm{E}-07$ \\
\hline 4 & $\mathrm{D}$ & NVT & NVD & SST/dist & SDHP/dist & & 5 & 504,28 & 41,30 & $8,15 \mathrm{E}-08$ \\
\hline 5 & $\mathrm{D}$ & NVD & STD & SST/dist & SDHP/dist & & 5 & 504,33 & 41,26 & $8,32 \mathrm{E}-08$ \\
\hline 6 & $\mathrm{D}$ & NVD & SST/dist & SDHP/dist & & & 4 & 504,61 & 38,97 & $7,05 \mathrm{E}-08$ \\
\hline 7 & $\mathrm{D}$ & STT & NVD & SST/dist & SDHP/dist & & 5 & 504,62 & 40,96 & $9,54 \mathrm{E}-08$ \\
\hline 8 & $\mathrm{D}$ & STT & NVD & STD & SDHP/dist & & 5 & 504,70 & 40,89 & $9,88 \mathrm{E}-08$ \\
\hline 9 & $\mathrm{D}$ & NVD & SST/dist & SDHP/dist & ID & & 8 & 505,05 & 46,53 & $1,88 \mathrm{E}-07$ \\
\hline 10 & $\mathrm{D}$ & NVT & STT & NVD & STD & SDHP/dist & 6 & 505,19 & 42,39 & $1,54 \mathrm{E}-07$ \\
\hline
\end{tabular}

NB: DL = degré de liberté, AIC = Akaike Information Criterion, Var. = Variable., D=Diamètre/Diameter/ Les formules utilisées sont référencées dans le Tableau 1. 


\section{DISCUSSION}

\section{Variabilité de la croissance du Moabi}

L'accroissement diamétrique moyen de $0,825 \pm 0,158 \mathrm{~cm} /$ an obtenu dans la présente étude serait spécifique aux arbres de plus de $100 \mathrm{~cm}$ de diamètre (Delphin et al., 2002). Mais, nous avons obtenu des arbres de 40 à 50 $\mathrm{cm}$ de diamètre avec cette performance. Ceci serait le fait de la perturbation due à l'exploitation forestière qui a contribué à ouvrir la canopée forestière, permettant ainsi aux jeunes arbres d'accéder facilement à la lumière. La différence significative d'accroissement entre les classes de diamètre obtenue, avec une proportion de variabilité expliquée de $52,4 \%$ en forêt tropicale perturbée peut s'expliquer par le fait que la croissance des arbres de Moabi n'est pas linéaire entre les petits et les grands diamètres respectivement en dessous et au-dessus de la canopée (Delphin et al., 2002; Moneye, 2012). Cependant, cette variabilité dans la croissance pourrait aussi être la conséquence de la compétition interspécifique entre les arbres centraux de Moabi et leurs voisins, ou de l'influence des paramètres environnementaux tels que l'accessibilité de l'éclairement solaire par le houppier des arbres, ou encore la disponibilité des ressources hydriques et des nutriments naturels du sol.

Pour le Moabi, le fait que la plus petite valeur d'accroissement moyen en diamètre $(0,38 \pm 0,42 \mathrm{~cm} / \mathrm{an})$ soit observée dans la classe « $\mathrm{ID}_{1} »$, c'est-à-dire chez les arbres dont les cimes sont entièrement ombragées et donc qui ne reçoivent pas de lumière, par opposition à la valeur d'accroissement diamétrique moyen la plus importante $(1,12 \pm$ $0,357 \mathrm{~cm} / \mathrm{an})$ enregistrée dans la classe « $\mathrm{ID}_{4}$ » des arbres entièrement exposés à la lumière verticalement et ombragés latéralement, indique clairement le comportement héliophile non-pionnier de l'espèce (Doucet et al., 2003) qui tolère ainsi l'ombre à tous les stades de son développement. L'évaluation de l'éclairement du houppier sur la base des moyennes d'accroissement diamétrique et des classes de statut social a révélé un effet significatif indiquant que l'environnement lumineux a une influence significative sur la croissance $\mathrm{du}$ Moabi avec une variance totale de l'accroissement expliquée par cet indice de compétition de $21,5 \%$. Ces résultats sont semblables à ceux obtenus pour le Sapelli par Moneye (2012) et pour le Moabi par Delphin et al. (2002).

En outre, le diamètre de référence, influence aussi significativement la croissance du Moabi et constitue un des principaux facteurs de variation de l'accroissement du diamètre ou de la surface terrière (Hérault et al., 2011). La variable "diamètre initial" serait à l'origine d'une forte variation de l'accroissement en diamètre du Moabi, avec une proportion de variabilité expliquée de 20,2\% ; Ceci s'apparente à celui observé par Moneye (2012) pour le Tali (25,7\%).

\section{Comportement du Moabi face aux indices de compétition indépendants et dépendants des distances}

L'expression de l'indice de densité locale NVD (nombre de voisins imposants par rapport au diamètre de l'arbre central) explique au mieux l'accroissement en diamètre $\left(R^{2}=33,10\right)$ et l'accroissement en surface terrière $\left(R^{2}=30,08\right)$ dans la présente étude. Ces valeurs sont meilleures que celles observées par Moneye (2012) qui a obtenu des indices de compétition utilisés dans notre site d'étude permettant d'expliquer $17,9 \%$ de la variance observée sur l'accroissement moyen annuel en diamètre du Sappeli. L'indice de densité locale STD (somme de surfaces terrières voisins imposants par rapport au diamètre de l'arbre central) qui explique le mieux l'accroissement en diamètre $\left(R^{2}=21,70\right)$ et l'accroissement en surface terrière $\left(\mathrm{R}^{2}=23,00\right)$ pour le Moabi s'apparentent le mieux à ceux que donnent Moneye (2012) dans les unités forestières d'aménagement 10041, 10042, 10044 (17,9\% de la variance observée sur l'accroissement moyen annuel en diamètre du Sappeli). Parmi les indices de compétition dépendant des distances étudiés (SST/dist, SSTr/dist et SDHP/dist), l'indice de concurrence STTr/Dist est la seule variable 
qui explique le mieux l'accroissement en diamètre $\left(\mathrm{R}^{2}=20,00\right)$; en outre, Bekono (2013) n'a observé qu'une corrélation de 5,9\% pour l'Assamela dans le même site d'étude.

\section{Principaux facteurs de variation de la croissance du Moabi}

Les fortes corrélations observées entre les facteurs de croissance et les variables explicatives de densité locale et de compétition montrent que les facteurs de croissance du Moabi correspondent aux variables de statut social des arbres centraux (D, ID), aux variables de statut social du peuplement (STD, SSTr/dist, NVD) et aux variables de densité locale du peuplement (STT, SDHP/dist, NVT, SST/dist). L'opposition observée entre les variables du statut social des arbres centraux (ID, D) et celles de densité locale du peuplement (NVT) et de densité locale du peuplement (STT, SST/dist) d'une part; et d'autre part ces variables de densité locale du peuplement et celles du statut social du peuplement (STD, NVD, SSTr/dist) et de variables de densité locale du peuplement (SDHP/dist) s'apparentent au résultats obtenus par Ndiaye et al. (2017) entre les variables de "densité", "richesse spécifique", "indice de Shannon" et "rendement" situés en abscisse positive et s'opposant à la variable diamètre située en abscisses négatives. Cette opposition traduit ainsi l'importance majeure de ces facteurs de croissance en foresterie car la compétition intervient dans de très nombreux modèles de croissance et de dynamique forestière (Prevosto, 2005). En outre, l'analyse factorielle des correspondances réalisée par Ndong et al. (2015) sur une matrice de 120 relevés et 38 espèces végétales pour définir les différents groupements végétaux dans six villages du Ferlo au Sénégal montre des axes $\mathrm{F} 1$ et $\mathrm{F} 2$ cumulés de $33 \%$ largement en deçà des $140,5 \%$ cumulés dans le cadre de cette étude. Ainsi, il apparaît que la croissance des espèces végétales ne peut se faire si on n'a pas une maîtrise parfaite de l'éclairement du houppier des arbres, c'est-à-dire de la quantité de lumière dont a besoin une plante pour réaliser sa photosynthèse (Prevosto, 2005).

\section{Prédictibilité de la croissance du Moabi}

L'évaluation de la performance par ordre décroissant (Tableaux 7 et 8 ) des régressions multiples sélectionnant les variables prédictives pertinentes de l'accroissement en diamètre et en surface terrière du Moabi à partir de la plus petite valeur de l'AIC permet de déduire que : la combinaison des indices de compétition dépendants et ceux indépendants des distances semblent être les plus performants à prédire l'accroissement diamétrique et en surface terrière du Moabi; les indices les plus pertinents, en tenant compte de l'analyse en composante multiple et des indices impliqués dans la modélisation de l'accroissement en diamètre et en surface terrière, sont $\mathrm{D}$, ID, NVT, NVD et STT. Par ailleurs, la combinaison prédisant le mieux l'accroissement diamétrique du Moabi comporte trois variables (deux indices indépendants de distance et deux indices dépendant des distances) avec un AIC de 83,51 . Pour l'accroissement en surface terrière, le meilleur modèle sera constitué des variables D, NVT, NVD, STD, SST/dist et SDHP/dist $(\mathrm{AIC}=503,49)$. En effet, ces modèles suffisamment plus complexes pour prendre en compte l'impact des changements environnementaux et suffisamment robustes pour fournir des prédictions fiables sur le comportement à long terme des forêts (Goreaud et al., 2007) sont d'autant plus urgent à développer à l'heure où l'aménagement forestier durable en zone tropicale n'est basé à ce jour que sur des simulations des tables de peuplement d'arbres qui sont en fait le résultat d'observations à long terme de l'évolution de peuplements de référence. Ces premiers outils présentent cependant, deux principaux inconvénients car ils ne rendent compte que de l'évolution moyenne des peuplements, et en général ils ne permettent pas de simuler différents types de 
sylviculture ou de nouveaux scénarios (Goreaud et al., 2005).

\section{Conclusion}

La mesure de la compétition pour les ressources ligneuses est longue et nécessite un appareillage spécialisé. Malgré les contraintes dues à la taille réduite de l'échantillon d'arbres retenus dans la présente étude et à l'absence de données de croissance sur le peuplement d'arbres concurrents ne permettant pas d'évaluer clairement l'effet progressif de la compétition et celui réellement engendré par la perturbation due à l'exploitation forestière sur la croissance du Moabi, il ressort tout de même que : au-delà des indices de concurrence indépendants et dépendants de la distance, il y a également des variables de prédiction de la croissance telles que le diamètre (D) et l'indice d'éclairement $\mathrm{du}$ houppier (ID) qui peuvent rentrer dans l'élaboration d'un modèle de prédiction efficient de son accroissement en diamètre et en surface terrière ; après analyses multivariées des différents indices de compétition, d'une manière générale les indices de concurrence qui sont les mieux corrélés aux facteurs de variation de sa croissance sont prioritairement ceux du statut social des arbres centraux (ID, D), de la densité locale du peuplement (STT, NVT) et du statut social du peuplement (STD, NVD) qui sont toutes des indices de densité indépendant de la distance; le meilleur modèle de prédiction de son accroissement en diamètre est constitué des variables explicatives SST/dist et SDHP/dist (indices dépendant des distances), NVD, NVD (indices de densité locale) et SDHP/dist (indice dépendant de la distance) ; le meilleur modèle de prédiction de son accroissement en surface terrière est constitué des variables explicatives D, NVT, NVD, STD, SSTr/dist et SDHP/dist. La présente étude est un précurseur dans l'élaboration future des modèles de croissance des essences dans les peuplements hétérogènes. Mais il se trouve que pour $\mathrm{y}$ parvenir, plusieurs facteurs environnementaux devront être pris en compte (compétition pour l'air ou pour la lumière, les nutriments du sol, la disponibilité de la ressource hydrique) en dehors de la compétition des arbres voisins par rapport aux arbres cibles. Ainsi, en développant cette réflexion jusqu'à son terme, on pourrait mieux comprendre la compétition que subissent les espèces et proposer des modèles de prédiction de leur croissance pour la gestion durable des forêts denses tropicales humides.

\section{CONFLITS D'INTERETS}

Les auteurs déclarent qu'ils n'ont aucun conflit d'intérêts.

\section{CONTRIBUTIONS DES AUTEURS}

ML a collecté les données de terrain, a rédigé le manuscrit, a conçu les cartes. KSB a contribué à leur lecture, à l'amélioration et à la validation du manuscrit.

\section{REMERCIEMENTS}

Les auteurs remercient la société d'exploitation forestière PALLISCO qui a mis à leur disposition le matériel roulant et les outils techniques pour réaliser cette étude.

\section{RÉFÉRENCES}

Agbo IR, Missihoun AA, Vihotogbe R, Assogbadjo EA, Ahanhanzo C, Agbangla C. 2017. Impacts des usages traditionnels sur la vulnérabilité de Detarium microcarpum Guill. \& Perr. (Caesalpiniaceae) dans le district phytogéographique Zou au Bénin (en Afrique de l'Ouest). International Journal of Biological and Chemical Sciences, 11(2): 730-743. DOI: https://dx.doi.org/10.4314/ijbcs.v11i2.16

Bekono K. 2013. Détermination de l'indice de concurrence au sein des peuplements de Pericopsis elata, (Harm) (Assamela) en forêt dense tropicale. Université de Dschang. Faculté d'agronomie et des sciences agricoles. Mémoire présenté en vue de l'obtention du Diplôme d'Ingénieur des Eaux, Forêts et Chasse. $77 \mathrm{p}$.

Blanc L, Flores O, Molino JF, Gourlet-Fleury S, Sabatier D. 2003. Diversité spécifique et regroupement d'espèces arborescentes 
en forêt guyanaise. Revue Forestière Française, 55: 131 - 146.

Daniels RF, Burkhart HE, Clason TR. 1986. A comparison of competition measures for predicting growth of loblolly pine trees. Canadian Journal of Forest Research, 16: $1230 \quad-1237 . \quad$ DOI: https://doi.org/10.1139/x86-218

Dawkins HC. 1958. The management of natural tropical high-forests with special reference to Uganda. Imperial Forestry Institute, University of Oxford. Institute Paper 34; 155.

Debroux L. 1998. L'aménagement des forêts tropicales fondé sur la gestion des populations d'arbres: l'exemple du Moabi (Baillonella toxisperma Pierre) dans la forêt du Dja, Cameroun. Thèse de Doctorat. Faculté Universitaire des Sciences agronomiques de Gembloux. Belgique, 283p.

Delphin M, Louppe D, Ingueza D. 2002. Fiche de Présentation du Moabi. CIFOR. CIRAD : Moabi ; 4

Detienne P, Oyono F, De Madron LD, Demarquez B, Nasi R. 1998. L'analyse de cernes : applications aux études de croissance de quelques essences en peuplements naturels de forêt dense africaine. CIFOR - Forêt Montpellier. Série FORAFRI. Document 15. CIRAD. 42p.

De Madron LD. 2003. Accroissement diamétrique du Bété et de l'Iroko. Note de recherche: Bois et Forêt des Tropiques, $275: 83-85$.

Dibong SD, Ndjouondo GP. 2014. Inventaire floristique et écologie des algues des rivières Kambo et Longmayagui de la zone humide de Douala (Cameroun). International Journal of Biological and Chemical Sciences, 8(6): 2561 - 2577. DOI: http://dx.doi.org/10.4314/ijbcs. v8i6.18

Doucet JL, Kouadio YL. 2007. Le Moabi, une espèce "phare» de l'exploitation forestière en Afrique Centrale. Revue Parcs et Réserves, 62(2). DOI : http://pallisco-

cifm.com/download/ParcsEtReserves62 moabi_juin2007.pdf
FAO. 2011. Situation des Forêts du Monde. FAO : Rome; 193.

Feteke F, Hubert D, Nkolong E. 2004. Plan d'Aménagement de l'UFA 10030. Document final. Pallisco. Cameroun, 168.

Franc A. 2008. Etudier et comprendre une forêt mélangée : une tâche difficile. Réflexions basées sur le développement des recherches en forêts boréale, tempérée et tropicale Étudier et comprendre une forêt mélangée : une tâche difficile. Réflexions basées sur le développement des recherches en forêts boréale, tempérée et tropicale. Revue Forestière Française, $\quad \mathbf{6 0}(2)$ : DOI : http://documents.irevues.inist.fr/bitstrea m/handle/2042/18134/129_138_FRANC .pdf? sequence $=1$

Gourlet-Fleury S. 1998. Indices de concurrence en forêt dense tropicale humide: étude de cas sur le dispositif sylvicole expérimental de Paracou (Guyane française). Annales des Sciences Forestières, 55(6): 623 - 653. DOI :

https://hal.inria.fr/file/index/docid/88322 6/filename/hal-00883226.pdf

Goreaud F, De Coligny F, Courbaud B, Dhote JF, Dreyfus PH, Perot T. 2005. La modélisation : un outil pour la gestion et l'aménagement en forêt. VertigO, Paris : Avancées Cinématographiques, 6(2): 112. DOI : http://hal.ird.fr/hal00016762/document

Goreaud F, Allain R, Courbaud B, Bieng M.A, Pérot T, Piroche JN. 2007. Simuler des peuplements de structures variées pour faciliter l'utilisation des modèles "arbre" spatialisés Revue Forestière Française, $\quad \mathbf{5 9}(2) . \quad$ DOI : http://documents.irevues.inist.fr/bitstrea m/handle/2042/9017/137_161.pdf?seque nce $=1$

Hérault B, Bachelot B, Poorter L, Rossi V, Bongers F, Chave J, Paine CET, Wagner F, Baraloto C. 2011. Functional traits shape ontogenetic growth trajectories of rain forest tree species. Journal of Ecology, 99: 1431-1440. DOI: 
http://vrossi.free.fr/DONNEES/HeraultJ Ecol11.pdf

Houllier F, Bouchon J, Birot Y. 1991. Modélisation de la dynamique des peuplements forestiers : état et perspectives. Revue Forestière Française, 33(2): 87-108. DOI : http://documents.irevues.inist.fr/bitstrea m/handle/2042/26197/RFF_1991_2_87.p df?se

Hill MO, Smith AJE. 1976. Principal component analysis of taxonomic data with multi-state discrete characters. Taxon, 25: 249-255. DOI: ftp://pbil.univlyon1.fr/pub/mac/ADE/ADE4/DocThem PDF/Thema2B.pdf

MINEF. 1999. Traitement des inventaires appliqué à la modélisation des aménagements (TIAMA). République du Cameroun: Manuel d'utilisation du logiciel. Ministère de l'Environnement et des Forêts. Direction des Forêts - Agence Canadienne de Développement International (projet 232/18027) Tecsult Inc., Québec.

Moneye JJ. 2012. Contribution à l'aménagement des forêts denses humides tropicales de l'est du Cameroun : détermination des indices de concurrence du Sapelli (Entandrophragma cylindricum Sprague Sprague) et du Tali (Erythrophleum suaveolens (Guill. \& Perr.) Brenan). Mémoire présenté en vue de l'obtention du Diplôme d'Études Supérieures Spécialisées/Master 2 en Aménagement et Gestion Intégrés des Forêts et Territoires Tropicaux, Université de Kinshasa, Kinshasa (Congo Démocratique). $218 \mathrm{p}$.

Moravie MA, Durand M, Houllier F. 1999. Ecological meaning and predictive ability of social status, vigour and competition indices in a tropical rain forest (India). Forest Ecology and Management, 131(1-3): 269-289. DOI : https://doi.org/10.1016/S03781127(98)00480-0
Ndiaye S, Charahabil MM, Ousmane N, Diatta M. 2017. Influence de la flore ligneuse associée dans la production des parcs à Anacardium occidentale L. dans la communauté rurale de Djibanar (Casamance/Sénégal). International Journal of Biological and Chemical Sciences, 11(2): 585 - 596. DOI: https://dx.doi.org/10.4314/ijbcs.v11i2.5

NDONG AT, Ndiaye O, Sagna MB, Diallo A, GALOP D, Guisse A. 2015. Caractérisation de la végétation ligneuse sahélienne du Sénégal: cas du Ferlo. International Journal of Biological and Chemical Sciences, 9(6): 2582 - 2594. DOI:

https://www.ajol.info/index.php/ijbcs/arti cle/view/135518/125025

Ofori DA. 2007. Milicia excelsa (Welw.) C.C.Berg. In Bois d'Euvre 1. Louppe D, Oteng-Amoako AA, Brink M (eds). Prota:

Timbers/Wageningen, The Netherlands: PROTA. DOI: http://database.prota.org/PROTAhtml/Mi licia\%20excelsa_Fr.htm

Parde J, Bouchon J. 1988. Dendrométrie (2 édn). ENGREF ; 328.

Picard N. 2007. Modélisation de la dynamique de forêts naturelles tropicales : quel niveau de description? Mémoire de synthèse en vue d'une candidature à une habilitation à diriger des recherches. Université Montpellier II. 100p.

Prevosto B. 2005. Les indices de compétition en foresterie : exemples d'utilisation, intérêts et limites. Revue Forestière Française, 57: 413 - 430. DOI http://documents.irevues.inist.fr/bitstrea $\mathrm{m} /$ handle/2042/5062/413_430.pdf?seque nce $=1$

Rondeux J. 1999. La Mesure des Arbres et des Peuplements Forestiers $\left(1^{\mathrm{e}}\right.$ edn). Les Presses agronomiques: Gembloux, Belgique ; 521 .

Steneker GA, Jarvis JM. 1963. A preliminary study to access competition in a white spruce-trembling aspen stand. Forest Chronicle, 39: 334 - 336. DOI : http://nofc.cfs.nrcan.gc.ca/bookstore_pdf s/30597.pdf 